\title{
Coronary artery fistula and bronchiectasis: case report and literature review
}

\author{
Liyuan Zhu ${ }^{1,2,3,4}$, Ling $\operatorname{Liu}^{1,2,3,4}$ \\ ${ }^{1}$ Department of Cardiovascular Medicine, the Second Xiangya Hospital, Central South University, Changsha, China; ${ }^{2}$ Research Institute of Blood \\ Lipid and Atherosclerosis, Central South University, Changsha, China; ${ }^{3}$ Modern Cardiovascular Disease Clinical Technology Research Center of \\ Hunan Province, Changsha, China; ${ }^{4}$ Cardiovascular Disease Research Center of Hunan Province, Changsha, China \\ Correspondence to: Ling Liu. Department of Cardiovascular Medicine, The Second Xiangya Hospital, Central South University, \#139 Middle Renmin \\ Road, Changsha 410011, China. Email: feliuling@csu.edu.cn.
}

\begin{abstract}
Coronary artery fistula (CAF) is characterized by abnormal communication between the main coronary artery and the cardiac chambers, pulmonary arteries, vena cava, and systemic arteries and veins, and is a rare cardiac malformation. Bronchiectasis involves remodeling and persistent dilatation of the bronchial airways. It can either be localized to one lung segment or diffused throughout the whole lung. The incidence of bronchiectasis has increased to 566 per 100,000 over the last decade. There is a $33 \%$ possibility that CAF and lung-related disease will co-occur. However, the merging mechanism of these two diseases has not been explored. We report a case of a 61-year-old female with bronchiectasis who was admitted to our hospital with dyspnea and ankle edema. The patient was diagnosed with coronary artery-pulmonary artery fistula by coronary angiography (CAG), which was performed to rule out the possibility of coronary artery stenosis. Using the keywords "coronary artery fistula" and "bronchiectasis" to search the inpatient system of our hospital, PubMed, and Web of Science, we found that these two diseases often occur due to abnormal cardiovascular and pulmonary airway connection. Previous studies also support that CAF can co-occur with bronchiectasis. Based on these findings, we explain the possible mechanism of this co-occurrence with the aim of improving diagnostic techniques and therapeutic methods.
\end{abstract}

Keywords: Coronary artery fistula (CAF); bronchiectasis; chest pain; case report

Submitted Dec 28, 2020. Accepted for publication Mar 04, 2021.

doi: 10.21037/apm-20-2608

View this article at: http://dx.doi.org/10.21037/apm-20-2608

\section{Introduction}

Coronary artery fistula (CAF) was first described in 1865 (1). $\mathrm{CAF}$ is characterized by abnormal communication between the main trunk or branch of the coronary artery and the heart cavity, pulmonary artery, coronary sinus, and vena cava. CAF is a common congenital cardiac malformation, but it is rarely acquired from trauma or infection (2). The clinical manifestation of CAF depends on shunt location and size. Fifty percent of patients with CAF have no clinical symptoms; thus, CAF is only observed upon physical examination when symptoms of myocardial ischemia are identified due to a coronary artery shunt, accompanied by typical chest angina, dyspnea, and limitations in daily activity (3).

Bronchiectasis was first described by Laennec in 1819 and defined as irreversible expansion and wall thickening of the bronchus. Bronchiectasis involves unrecoverable abnormal expansion of the local bronchus and destruction of elastic and muscle tissue in the bronchial wall, leading to abnormal and persistent bronchial expansion. Meanwhile, expanded bronchial arteries form pseudo-hemangiomas composed of destroyed blood vessel elastic fibers and uneven blood vessel thickness, causing rupture and bleeding. The clinical manifestations of bronchiectasis include cough, purulent sputum, and recurrent hemoptysis $(4,5)$.

There is a $33 \%$ possibility that CAF and lung-related disease will co-occur (6). However, the merging mechanism 


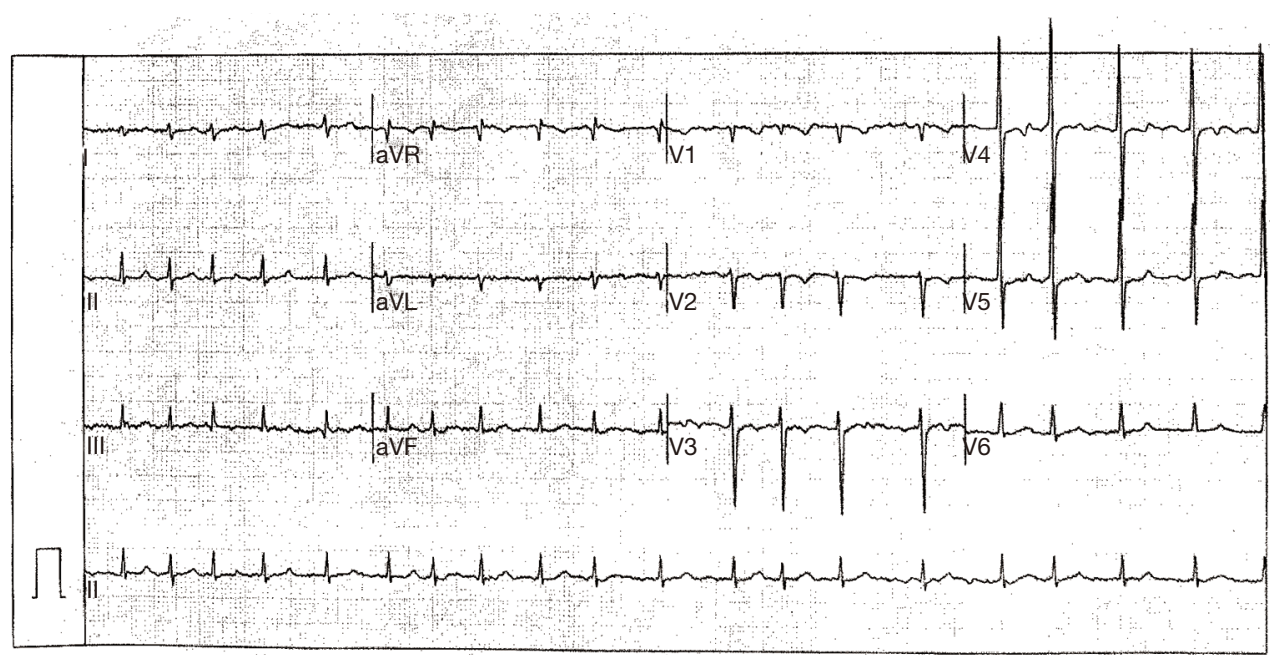

Figure 1 Electrocardiograph on admission showing atrial fibrillation with a rapid atrioventricular rate.

of these two diseases has not been explored. This article reported a 61-year-old female admitted to hospital due to chest pain, dyspnea, and edema in both lower limbs. The patient was diagnosed with coronary artery-pulmonary artery fistula with bronchiectasis. Moreover, we briefly reviewed patients with co-existing $\mathrm{CAF}$ and bronchiectasis from 2011 to 2018 using data from our hospital's inpatient system. In addition, we summarized the number of cases and types of CAF, as well as the history and location of bronchiectasis, by searching combined case reports in PubMed and Web of Science. We performed a literature review of these cases to explore the possible co-occurrence mechanism of CAF and bronchiectasis. Our observations could aid clinical diagnosis and treatment of patients with co-existing CAF and bronchiectasis.

We present the following article in accordance with the CARE reporting checklist (available at http://dx.doi. org/10.21037/apm-20-2608).

\section{Methods}

We searched the inpatient system of the Second Xiangya Hospital of Central South University from January 2011 to December 2018. One hundred eighty-three patients were diagnosed with CAF. Among them, 5 patients were also diagnosed with co-existing bronchiectasis. In addition, 6,434 patients were diagnosed with bronchiectasis. Among them, 5 patients were also diagnosed with co-existing CAF. The 5 selected and matched patients from the two cohorts (a total of 5 patients with co-existing CAF and bronchiectasis) were included in the study.

We also searched PubMed and Web of Science using the keywords "coronary artery fistula" and "bronchiectasis," the study languages were limited to English and Chinese, and only studies on diagnosis and treatment were included. A total of 14 studies published between 1999 and 2019 were retrieved. A total of 16 patients with co-existing $\mathrm{CAF}$ and bronchiectasis were identified.

\section{Case presentation}

A 61-year-old female patient was admitted to the Department of Cardiology of the Second Xiangya Hospital of Central South University on December 26, 2018, with symptoms of chest pain, dyspnea, and lower-limb edema for 2 years. These symptoms were induced and aggravated by walking and dressing, and were accompanied by paroxysmal nocturnal dyspnea in the recent 2 months. The patient had bronchiectasis for 10 years and massive hemoptysis 8 years ago, for which she did not receive treatment. Laboratory tests showed an $\mathrm{N}$-terminal pro-brain natriuretic peptide concentration of $1,688.49 \mathrm{pg} / \mathrm{mL}$.

On admission, electrocardiography showed atrial fibrillation with a rapid ventricular rate (Figure 1). Echocardiographic findings showed left atrial enlargement $(44 \mathrm{~mm})$, decreased left ventricular systolic function (ejection fraction: $50 \%$, fractional shortening: $26 \%$ ), arrhythmia and tachycardia, a small amount of pericardial effusion, and no signs of abnormal wall motion. Chest radiography and chest computerized tomography revealed pulmonary 

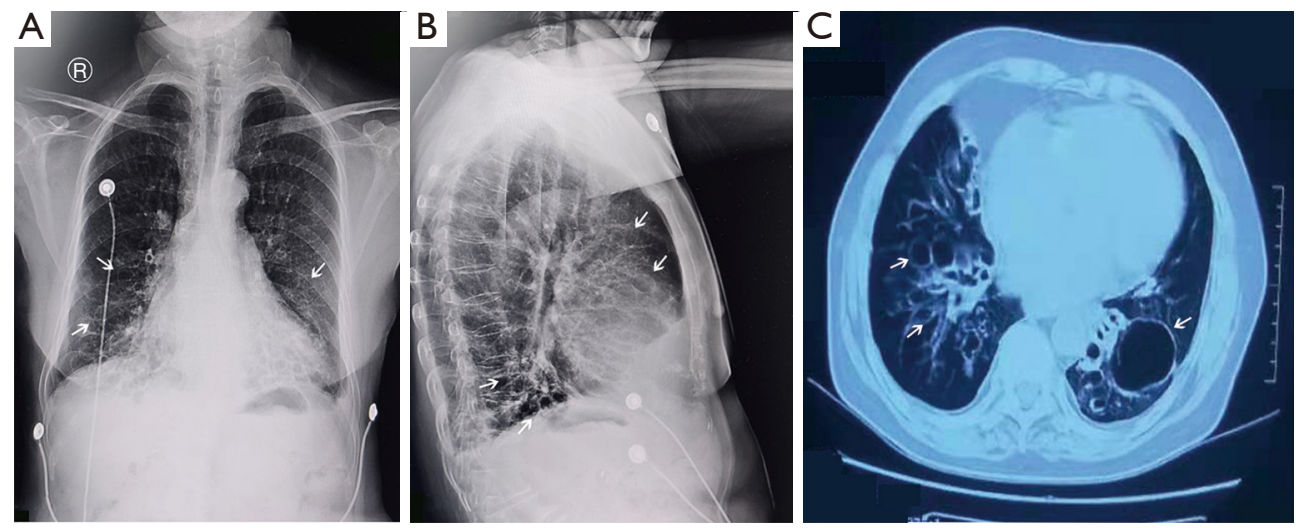

Figure 2 Pulmonary radiological images on admission. Bronchiectatic changes in the bilateral lower lobes were identified by chest radiography $(\mathrm{A}, \mathrm{B})$ and computed tomography $(\mathrm{C})$. Solid arrow: location of bronchiectasis.

infection and bronchiectasis in both lower lobes and a small amount of pleural effusion with pleural thickening (Figure 2). Pulmonary function tests showed severe mixed ventilatory dysfunction.

After admission, the patient was administered symptomatic and supportive treatment. Quinamycin tablets were given for anti-infection. The symptoms of chest distress, dyspnea, and edema slightly improved after 1 week. $\mathrm{N}$-terminal pro-brain natriuretic peptide concentration decreased to $792.00 \mathrm{pg} / \mathrm{mL}$. The patient underwent coronary angiography (CAG) to confirm coronary artery stenosis. CAG revealed an unusually dilated, tortuous, anomalous vessel, CAF, arising from the proximal left circumflex (LCX) to the right. The CAF had three branches to supply the right lung (Figure $3 A, B, C$, Supplementary material of Videos 1,2). Considering that fistula and atrial fibrillation with a rapid ventricular rate further led to acute heart failure development in this patient, treatment aimed to control ventricular rate and eliminate the etiology of increased left ventricular preload caused by the CAF. There was no distal blood flow of the CAF after transcatheter coil embolization (Figure 3D and Supplementary material of Video 3). Chest pain and dyspnea subsided over a 6-month follow-up period.

All procedures performed in studies involving human participants were in accordance with the ethical standards of the institutional and/or national research committee(s) and with the Helsinki Declaration (as revised in 2013). Written informed consent was obtained from the patient for publication of this case report and any accompanying images.

\section{Hospitalized patients with CAF and bronchiectasis from 2011 to 2018}

The keywords "coronary artery fistula" and "bronchiectasis" were used to search the inpatient system of the Second Xiangya Hospital of Central South University from January 2011 to December 2018. We summarized 183 patients with $\mathrm{CAF}$ and 6,434 patients with bronchiectasis. One female and four males were selected with an average age of 62.5 years. Patients were admitted to hospital with chest tightness, chest pain, or dyspnea. Inpatient departments included respiratory departments $(2 / 5)$ and cardiovascular departments (3/5). CAF was diagnosed by CAG (3/5), computed tomography (1/5), or color Doppler ultrasonography (1/5). It is worth noting that one case of CAF co-occurred with congenital heart disease. The heart murmur was not consistently affected by shunt site or size. Most patients had a history of bronchiectasis for many years. Patients had bronchial expansion and infection in the left lower lobe of the lung with pleural effusion (2/5) or pleural thickening (1/5). Pulmonary function tests indicated different extents of ventilatory dysfunction. Conservative treatment (3/5) with drugs or elective surgery (2/5) was often adopted considering presence of severe pulmonary disease or old age (Table 1).

\section{Literature review of PubMed and Web of Science from 1999 to 2019}

We used the keywords "coronary artery fistula" and "bronchiectasis" to search PubMed and Web of Science. 

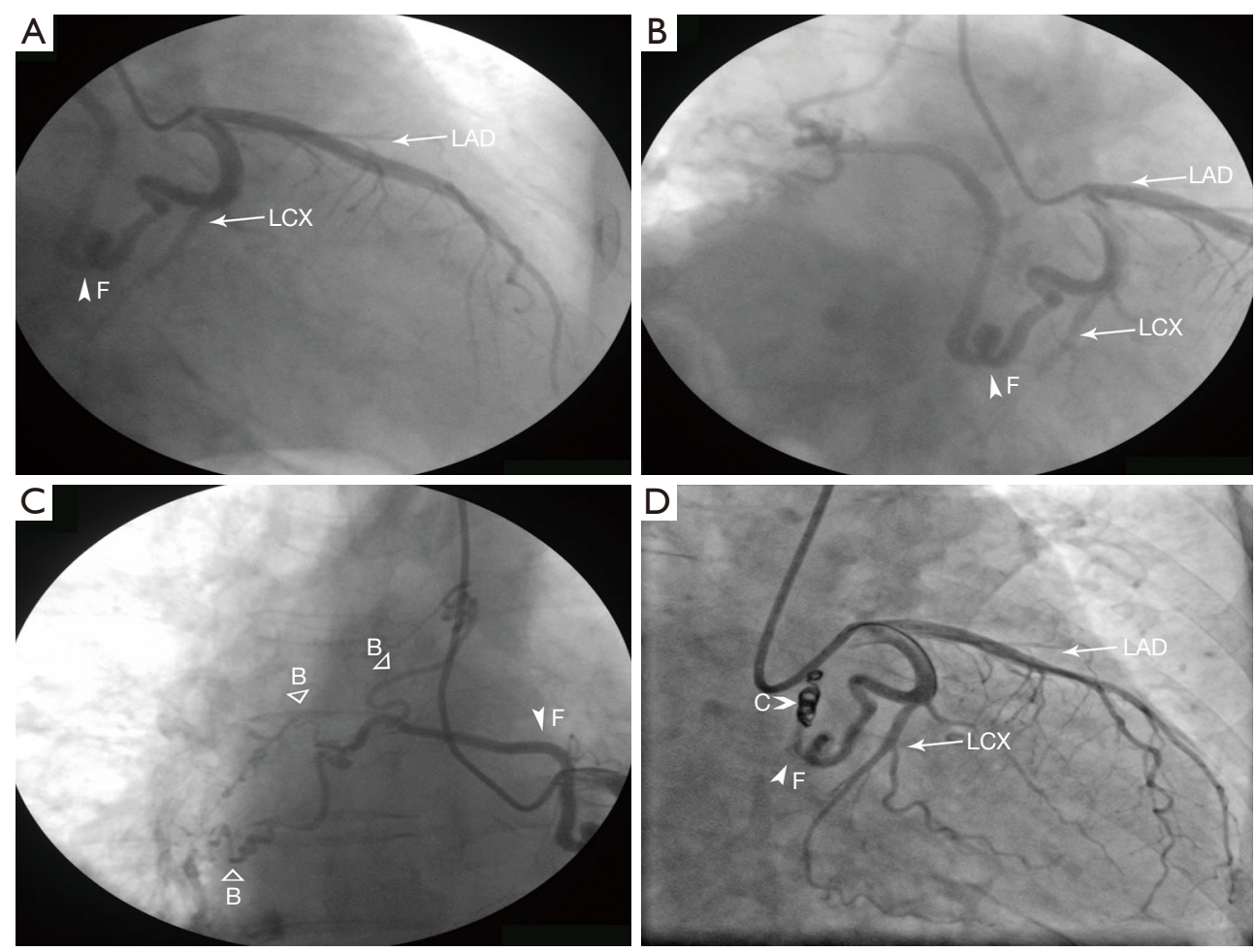

Figure 3 Coronary angiography images of CAF. The unusually dilated, tortuous, anomalous CAF is observed from the proximal LCX to the right (A,B). The CAF gave off three branches to supply the right lung (C). The CAF was closed without distal blood flow by transcatheter coil embolization (D). Short solid arrow with "F": location of CAF; short hollow arrow with "B": branches of CAF; short solid arrow with "C": location of coil embolization. CAF, coronary artery fistula; LCX, left circumflex artery; LAD, left anterior descending artery.

Fourteen studies were identified between 1999 and 2019, including studies on a total of 16 patients who completed diagnosis and treatment. The average age of patients was 61.8 years. Two patients with cystic fibrosis were $<40$ years of age. Patients with CAF combined with bronchiectasis had clinical symptoms, such as chest pain (11/16) and/ or dyspnea $(5 / 16)$. Three patients had symptoms of hemoptysis. All patients were diagnosed with CAF using CAG. Bronchiectasis was located at the lower lobe of the left lung (9/16). Patients underwent interventional therapy for CAF or surgery for complications (12/16). Conservative treatment or medical treatment (4/16) was adopted when patients were intolerable to surgery. Dyspnea and other symptoms improved after follow up (Table 2) (7-20).

\section{Discussion}

At present, no large-scale clinical investigations or animal experiments have investigated the mechanism of CAF combined with bronchiectasis; thus, further research is needed. The detection rate of CAF using CAG is $0.1-0.2 \%$ (21), and CAF accounts for $0.27-0.4 \%$ of all congenital heart disease cases $(22,23)$. We report a unique case of coronary artery-pulmonary artery fistula with bronchiectasis. This case helps us to understand the abnormal hemodynamics of CAF and provides a platform for further understanding its etiology and pathogenesis.

The congenital CAF is related to the vascular sinusoid cavity formed by cardiac endothelial cells during the embryonic period. The sinusoid cavity between the normally developed myocardial trabeculae gradually shrinks into small tubes as an individual grows, forming a regular coronary vascular system. Coronary arteries grow from the aortic root and are connected to the capillary network on the cardiac surface (2). With some developmental abnormalities, the sinusoid cavity persists and does not shrink during the growth, forming abnormal branches between coronary arteries and the cardiac cavity (24). Moreover, some cases of CAF are acquired from surgical trauma, coronary artery bypass grafting, CAG, 


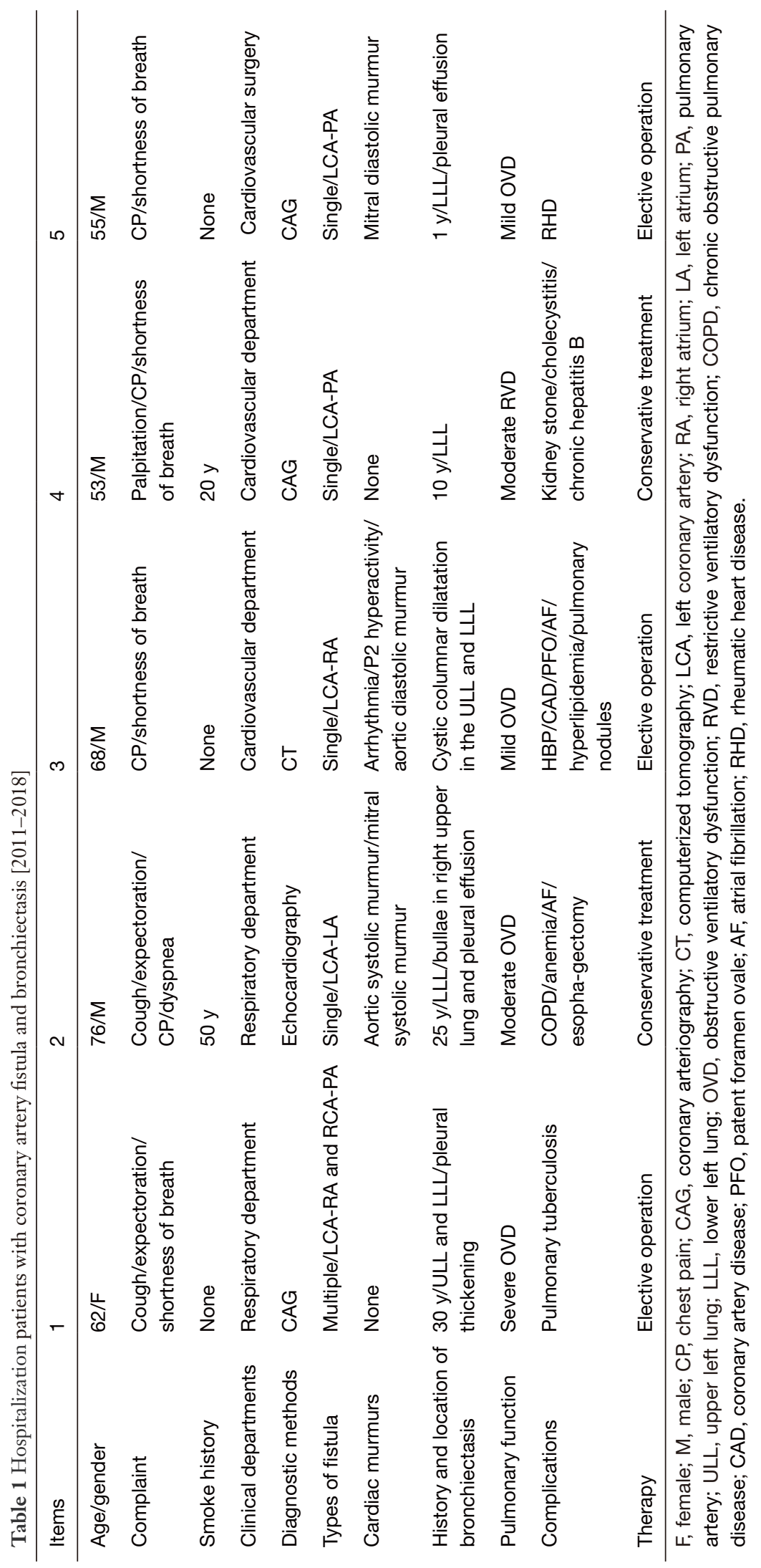




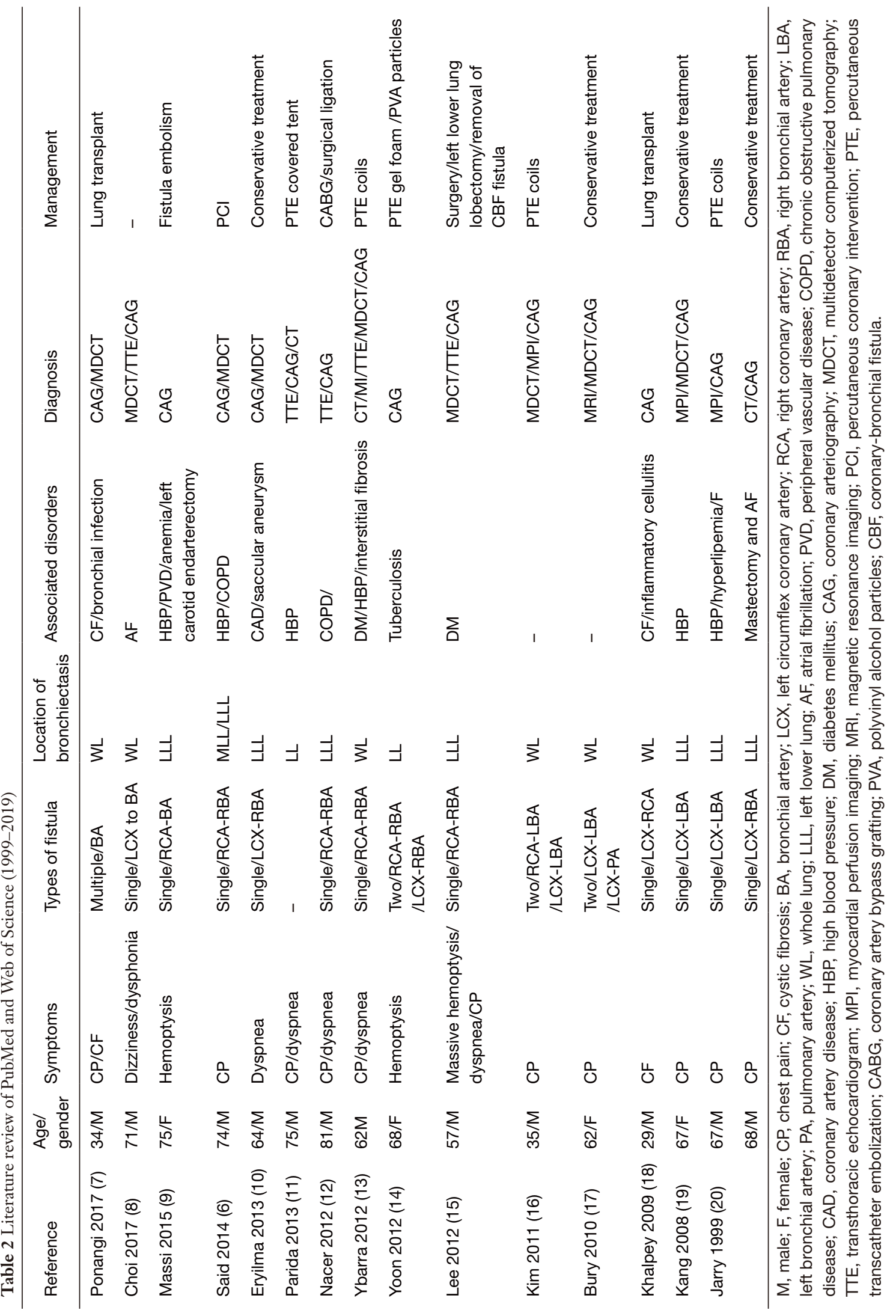


atherosclerosis, acute myocardial infarction, or permanent pacemaker implantation (25).

Some scholars have suggested that the complications of CAF and bronchiectasis may be related to pressure differences between heart and lung. Coronary arteries usually originate from the aorta and contain high-pressure blood flow, providing blood to the heart, in both the systolic and diastolic states. When there is a blood pressure gradient between coronary artery and the pulmonary arterial circulation, fistula flow occurs from high pressure to low pressure. The "coronary blood theft" phenomenon of the myocardium in patients with CAF occurs when coronary artery pressure increases, such as in aortic valve stenosis, or when pulmonary artery pressure decreases, such as in pulmonary atresia, Takayasu's arteritis, or chronic obstructive pulmonary disease (COPD) (26-30). Persistent infection and inflammation dilate bronchial arteries (31), leading to decreased resistance between the coronary circulation and the pulmonary circulation. When there is a pressure difference or when the body's demand or compensatory demand increases, the connection between the coronary artery and the pulmonary artery or bronchial artery reopens, forming a fistula (32). Most patients with COPD present with pulmonary hypertension combined with bronchiectasis, resulting in a low blood oxygen concentration. The body needs more lobes to integrate when breathing or exercising. Active peptides, including endothelin-1, in blood vessels are mostly secreted by endothelial cells, smooth muscle cells, and airway endothelial cells, and they exert strong vasocontraction (33). Endothelin-1 may stimulate initially closed non-functional outer pericardial coronary arteries and build functional connections with other organs, forming a functional pathway.

We believe that the systemic collateral supply in patients with pulmonary hypertension forms both bronchial and/ or non-bronchial systemic circulations. We speculate LCX artery fistula combined with bronchiectasis in our case for the following reasons. First, the patient had severe bronchiectasis in the lower part of the lung for 10 years, bulla in the right lung (Figure 2), and pulmonary hypertension. This may have led to insufficient oxygen supply to the lung parenchyma, and hypoxia may have induced new blood vessel formation in the lungs. Second, bronchiectasis is prone to co-infection, and repeated inflammatory conditions may also induce angiogenesis or dilatation. Third, the closed coronary sinusoidal space in juvenile development is only a physiological closure that can functionally open and form a fistula under conditions of pathological hypoxia and inflammation. Fourth, the LCX artery had a more prolonged course that extended behind the pulmonary artery compared with the right circumflex artery and left anterior descending vessels in our case, thus increasing the chances of developing a fistula from the LCX artery. Similarly, it has the possibility of fistula formation from the coronary artery to the bronchial artery combined with bronchiectasis, as reviewed in Table 2 in the bronchial system. We speculate that coronary artery-bronchial artery fistula may occur due to recurrent inflammation associated with bronchiectasis and secondary angiogenesis (which forms new connections) or increased vasodilation caused by local inflammation and opening of the existing latent fistula.

With advancements in diagnosis and treatment in recent years, whole-exome sequencing suggests that CAF and bronchiectasis may co-occur. In the selected cases, two young patients with cystic fibrosis were diagnosed with CAF during hospitalization due to severe bronchiectasis $(7,10)$. Cystic fibrosis is a common cause of bronchiectasis. In cases of abnormal coronary vascular malformation, abnormal mutations in the cystic fibrosis transmembrane conductance regulator (CFTR) gene were identified by exon sequencing (34). CFTR is a common gene locus mutation abnormality in cystic fibrosis, and mice with CFTR mutations are susceptible to respiratory disease (35). CFTR induces ectopic production of vascular epidermal growth factors, which contribute to new blood vessel formation (36). We speculate that this could lead to cystic fibrosis with coronary arteries of an abnormal origin and shape. In addition, patients with bronchiectasis are accompanied by diffuse inflammation, and inflammation triggers formation of new blood vessels. Therefore, when patients with cystic fibrosis experience chest tightness and pain, they should be alerted to the possibility of the CAF "stealing blood," which leads to chest tightness and pain.

The diagnostic technology of CAF combined with bronchiectasis is also being updated. In the past, CAG was the diagnostic standard for CAF. With improvements in non-invasive examination technology, it may be possible to consider computed tomography angiography (CTA) to diagnose CAF. It was regrettable that this patient did not undergo CTA of coronary artery because she had atrial fibrillation with a rapid ventricular rate, and the vascular condition around the bronchial arteries had no chance to show clearly. Although CTA is not as timely and accurate as CAG, three-dimensional reconstructed images obtained using CTA can show anatomical structures and complex 
lesions around nearby arteries, contributing to the diagnosis of CAF and identifying abnormal connections with peripheral blood vessels. CTA can also analyze whether bronchiectasis is combined with bronchial artery thickening and hypertrophy.

Treatment of patients with CAF usually involves occlusion technology. The prognosis of patients is relatively stable and safe when the treatment regime considers any comorbid symptoms. Patients with bronchiectasis often have severe respiratory infection, poor cardiopulmonary function, and obstructed ventilation, which may expand the contraindications to surgery. Taking conservative treatment with drugs could eliminate inflammation. The patient reported in this article underwent CAF closure surgery after respiratory system symptoms were controlled, and clinical symptoms, such as chest tightness and dyspnea, improved significantly. Asymptomatic patients with CAF may be misdiagnosed without cardiovascular imaging examination in other departments. Other patients with CAF are admitted to the Department of Respiratory with hemoptysis due to bronchiectasis. If patients combined with secondary and persistent hemoptysis after bronchial artery embolization, clinicians should be aware of the possibility of CAF and take suitable treatment to prevent disease progression.

In general, as a rare cardiovascular disease, CAF often co-occurs with bronchiectasis. Careful consideration should be given to patients' clinical symptoms and their general conditions during diagnosis and treatment. With the development of precision medicine and popularization of gene-sequencing technology, targeted prevention and medical treatment should be performed to prevent complications over time, such as congestive heart failure and coronary aneurysmal rupture, and to improve patient prognosis and quality of life.

\section{Acknowledgments}

We thank Professor Huijun Ye for his assistance during this study. We thank Elixigen Corporation (service@elixigen. com) for language editing of this manuscript.

Funding: The study was supported by the National Natural Science Foundation of China [grant numbers 81270956 and 81470577] and the Fundamental Research Funds for the Central Universities of Central South University [grant numbers 2020zzts882].

\section{Footnote}

Reporting Checklist: The authors have completed the CARE reporting checklist. Available at http://dx.doi.org/10.21037/ apm-20-2608

Conflicts of Interest: Both authors have completed the ICMJE uniform disclosure form (available at http://dx.doi. org/10.21037/apm-20-2608). The authors have no conflicts of interest to declare.

Ethical Statement: The authors are accountable for all aspects of the work in ensuring that questions related to the accuracy or integrity of any part of the work are appropriately investigated and resolved. All procedures performed in studies involving human participants were in accordance with the ethical standards of the institutional and/or national research committee(s) and with the Helsinki Declaration (as revised in 2013). Written informed consent was obtained from the patient.

Open Access Statement: This is an Open Access article distributed in accordance with the Creative Commons Attribution-NonCommercial-NoDerivs 4.0 International License (CC BY-NC-ND 4.0), which permits the noncommercial replication and distribution of the article with the strict proviso that no changes or edits are made and the original work is properly cited (including links to both the formal publication through the relevant DOI and the license). See: https://creativecommons.org/licenses/by-nc-nd/4.0/.

\section{References}

1. Krause W. Uberden Ursprung einer. Akzessorischen A. Coronaria aus der A. Pulmonalis. Z Ratl Med 1865;24:225-7.

2. Yun G, Nam TH, Chun EJ. Coronary Artery Fistulas: Pathophysiology, Imaging Findings, and Management. Radiographics 2018;38:688-703.

3. Challoumas D, Pericleous A, Dimitrakaki IA, et al. Coronary arteriovenous fistulae: a review. Int $\mathrm{J}$ Angiol 2014;23:1-10.

4. Polverino E, Goeminne PC, McDonnell MJ, et al. European Respiratory Society guidelines for the management of adult bronchiectasis. Eur Respir J 
2017;50:1700629.

5. Smith MP. Diagnosis and management of bronchiectasis. CMAJ 2017;189:E828-35.

6. Said SA, Oortman RM, Hofstra JH, et al. Coronary arterybronchial artery fistulas: report of two Dutch cases with a review of the literature. Neth Heart J 2014;22:139-47.

7. Ponangi UP, Dembo L, Musk M. Multiple Coronary to Bronchial Artery Fistulas Detected During Workup of a Young Patient with Cystic Fibrosis and Bronchiectasis for Lung Transplant. EC Cardiology 2017;3:228-31.

8. Choi HJ, Kim HW, Kim DY, et al. Surgical Management of a Coronary-Bronchial Artery Fistula Combined with Myocardial Ischemia Revealed by (13)N-Ammonia Positron Emission Tomography. Korean J Thorac Cardiovasc Surg 2017;50:220-3.

9. Massi F, Muretti M, Coradduzza E, et al. Myocardial infarction and rupture after bronchial artery embolization. Ann Thorac Surg 2015;99:1051-3.

10. Eryilmaz U, Gungor H, Uyar S, et al. Circumflex-tobronchial artery fistula with saccular aneurysm. Postepy Kardiol Interwencyjnej 2013;9:296-7.

11. Parida AK, Lodh M, Sharma JS, et al. Coronary Bronchial Steal. Journal of Case Reports 2013;3:39-43.

12. Nacar AB, Yorgun H, Tuncer C. Right coronary-tobronchial artery fistula on the contralateral side of coronary stenosis. Turk Kardiyol Dern Ars 2012;40:196.

13. Ybarra LF, Ribeiro HB, Hueb W. Coronary to bronchial artery fistula: are we treating it right? J Invasive Cardiol 2012;24:E303-4.

14. Yoon JY, Jeon EY, Lee IJ, et al. Coronary to bronchial artery fistula causing massive hemoptysis in patients with longstanding pulmonary tuberculosis. Korean J Radiol 2012;13:102-6.

15. Lee WS, Lee SA, Chee HK, et al. Coronary-bronchial artery fistula manifested by hemoptysis and myocardial ischemia in a patient with bronchiectasis. Korean J Thorac Cardiovasc Surg 2012;45:49-52.

16. Kim SK, Kim HW, Kim PJ, et al. Concomitant left and right coronary to bronchial artery fistulae resulting in myocardial ischaemia. Eur J Cardiothorac Surg 2011;39:278.

17. Bury RW, Wojciuk J, Goode GK. Multiple coronary artery fistulae associated with bronchiectasis: rarity or recognized phenomenon? Tex Heart Inst J 2010;37:380-1.

18. Khalpey Z, Camp P Jr, Jaklitsch MT. Left circumflex to bronchial artery fistula. Ann Thorac Surg 2009;88:303.

19. Kang WC, Moon C 2nd, Ahn TH, et al. Identifying the course of a coronary-bronchial artery fistula using contrast- enhanced multi-detector row computed tomography. Int J Cardiol 2008;130:e125-8.

20. Jarry G, Bruaire JP, Commeau P, et al. Coronary-tobronchial artery communication: report of two patients successfully treated by embolization. Cardiovasc Intervent Radiol 1999;22:251-4.

21. Darwazah AK, Hussein IH, Hawari MH. Congenital circumflex coronary arteriovenous fistula with aneurysmal termination in the pulmonary artery. Tex Heart Inst J 2005;32:56-9; discussion 58-9.

22. Cebi N, Schulze-Waltrup N, Fromke J, et al. Congenital coronary artery fistulas in adults: concomitant pathologies and treatment. Int J Cardiovasc Imaging 2008;24:349-55.

23. Schumacher G, Roithmaier A, Lorenz HP, et al. Congenital coronary artery fistula in infancy and childhood: diagnostic and therapeutic aspects. Thorac Cardiovasc Surg 1997;45:287-94.

24. Rangel-Abundis A, Munoz-Castellanos L, Marin G, et al. Morphofunctional correlation in congenital anomalies of the coronary arteries. I. Coronary artery fistulas. Arch Inst Cardiol Mex 1994;64:161-74.

25. Chiu SN, Wu MH, Lin MT, et al. Acquired coronary artery fistula after open heart surgery for congenital heart disease. Int J Cardiol 2005;103:187-92.

26. Koenig PR, Kimball TR, Schwartz DC. Coronary artery fistula complicating the evaluation of Kawasaki disease. Pediatr Cardiol 1993;14:179-80.

27. Said SA, van der Werf T. Acquired coronary cameral fistulas: are these collaterals losing their destination? Clin Cardiol 1999;22:297-302.

28. Ercan E, Tengiz I, Yakut N, et al. Takayasu's arteritis with multiple fistulas from three coronary arteries to lung paranchima. Int J Cardiol 2003;88:319-20.

29. Gasparovic H, Novick W, Anic D, et al. Iatrogenic coronary artery fistula in a patient with a single coronary artery. Thorac Cardiovasc Surg 2002;50:109-11.

30. Liang CD, Ko SF, Huang CF, et al. Echocardiographic evaluation of coronary artery fistula in pediatric patients. Pediatr Cardiol 2005;26:745-50.

31. Byun SS, Park JH, Kim JH, et al. Coronary CT findings of coronary to bronchial arterial communication in chronic pulmonary disease. Int J Cardiovasc Imaging 2015;31 Suppl 1:69-75.

32. Nepal S, Annamaraju P. Coronary Arteriovenous Fistula. StatPearls. Treasure Island, FL, 2021.

33. Öcal S, Portakal O, Öcal A, et al. Factors associated with pulmonary hypertension and long-term survival in bronchiectasis subjects. Respir Med 2016;119:109-14. 
34. Hekim N, Batyraliev T, Trujillano D, et al. Whole Exome Sequencing in a Rare Disease: A Patient with Anomalous Left Coronary Artery from the Pulmonary Artery (BlandWhite-Garland Syndrome). OMICS 2016;20:325-7.

35. Flume PA, Chalmers JD, Olivier KN. Advances in

Cite this article as: Zhu L, Liu L. Coronary artery fistula and bronchiectasis: case report and literature review. Ann Palliat Med 2021;10(7):8403-8412. doi: 10.21037/apm-20-2608 bronchiectasis: endotyping, genetics, microbiome, and disease heterogeneity. Lancet 2018;392:880-90.

36. Martin C, Coolen N, Wu Y, et al. CFTR dysfunction induces vascular endothelial growth factor synthesis in airway epithelium. Eur Respir J 2013;42:1553-62. 